\title{
Ground state solution for a Kirchhoff problem with exponential critical growth *
}

\author{
Giovany M. Figueiredo \\ Universidade Federal do Pará, Faculdade de Matemática, \\ CEP 66075-110, Belém-PA, Brasil \\ giovany@ufpa.br \\ Uberlandio B. Severo \\ Universidade Federal do Paraíba, Departamento de Matemática, \\ CEP 58051-900, João Pessoa-PB, Brasil \\ uberlandio@mat.ufpb.br
}

\begin{abstract}
We establish the existence of a positive ground state solution for a Kirchhoff problem in $\mathbb{R}^{2}$ involving critical exponential growth, that is, the nonlinearity behaves like $\exp \left(\alpha_{0} s^{2}\right)$ as $|s| \rightarrow \infty$, for some $\alpha_{0}>0$. In order to obtain our existence result we used minimax techniques combined with the TrudingerMoser inequality.
\end{abstract}

2010 Mathematics Subject Classification: 35J20, 35J25, 35J60, 35Q60.

Keywords and phrases: Kirchhoff problem, exponential critical growth, ground state solution.

\section{Introduction}

This work is concerned with the existence of a positive ground state solution for a nonlocal Kirchhoff problem of the type

$$
\left\{\begin{aligned}
-m\left(\|u\|^{2}\right) \Delta u & =f(x, u) & & \text { in } \quad \Omega, \\
u & =0 & & \text { on } \quad \partial \Omega,
\end{aligned}\right.
$$

where $\Omega$ is a smooth bounded domain in $\mathbb{R}^{2},\|u\|^{2}:=\int_{\Omega}|\nabla u|^{2} \mathrm{~d} x$ is the norm of the gradient in $W_{0}^{1,2}(\Omega), m: \mathbb{R}_{+} \rightarrow \mathbb{R}_{+}$and $f: \Omega \times \mathbb{R} \rightarrow \mathbb{R}$ are continuous functions that satisfy some appropriate conditions and they will be stated later on.

* Research partially supported by the National Institute of Science and Technology of Mathematics INCT-Mat, CNPq grants 308339/2010-0 and 454749/2011-2. 
Problem $(P)$ is called nonlocal because of the term $m\left(\|u\|^{2}\right)$ which implies that the equation in $(P)$ is no longer a pointwise identity. As we will see later the presence of the term $m\left(\|u\|^{2}\right)$ provokes some mathematical difficulties which makes the study of such a class of problems particularly interesting. Moreover, equation (P) has a physical appeal. The main motivation to study problem $(P)$ is due to the work of Kirchhoff [10] in which, in 1883, he studied the hyperbolic equation

$$
\rho \frac{\partial^{2} u}{\partial t^{2}}-\left(\frac{P_{0}}{h}+\frac{E}{2 L} \int_{0}^{L}\left|\frac{\partial u}{\partial x}\right|^{2} \mathrm{~d} x\right) \frac{\partial^{2} u}{\partial x^{2}}=0,
$$

that extends the classical D'Alembert wave equation, by considering the effects of the changes in the length of the strings during the vibrations. The parameters in equation (1.1) have the following meanings: $L$ is the length of the string, $h$ is the area of cross-section, $E$ is the Young modulus of the material, $\rho$ is the mass density and $P_{0}$ is the initial tension. In fact, $(P)$ can be seen as a stationary version of the following evolution problem:

$$
\left\{\begin{aligned}
\frac{\partial^{2} u}{\partial t^{2}}-m\left(\|u\|^{2}\right) \Delta u & =f(x, u) & & \text { in } \quad \Omega \times(0, T), \\
u & =0 & & \text { on } \quad \partial \times(0, T), \\
u(x, 0) & =u_{0}(x) & & \text { in } \quad \Omega, \\
\frac{\partial u}{\partial t}(x, 0) & =u_{1}(x) & & \text { in } \quad \Omega,
\end{aligned}\right.
$$

which have called the attention of several researchers mainly after the work of Lions [15], where a functional analysis approach was proposed to study it. We mention that nonlocal problems also appear in other fields, for example, biological systems where the function $u$ describes a process which depends on the average of itself (for example, population density), see for instance [2, 3, and its references.

In this paper, we are also interested in a borderline case of the Sobolev imbedding theorems, commonly known as the Trudinger-Moser case. When $n=2$, clearly the Sobolev exponent $2^{*}$ becomes infinite and $W^{1,2}(\Omega) \hookrightarrow L^{q}(\Omega)$ for $1 \leq q<\infty$ but $W^{1,2}(\Omega) \hookrightarrow L^{\infty}(\Omega)$. To fill this gap, at least in the case where $\Omega$ is a bounded domain, using the Dirichlet norm $\|\nabla u\|_{2}$ (equivalent to the Sobolev norm in $W_{0}^{1,2}(\Omega)$ ) and replacing the target Lebesgue space by an Orlicz space, N. Trudinger [19] proved that there exists $\alpha>0$ such that $W_{0}^{1,2}(\Omega)$ is embedded into the Orlicz space $L_{\phi_{\alpha}}(\Omega)$ determined by the Young function $\phi_{\alpha}(t)=\exp \left(\alpha t^{2}\right)-1$. This result had many generalizations, extensions and applications in recent years. In the first direction, it was sharpened by J. Moser [18, who found the best exponent $\alpha$ and in particular he proved the following result:

Moser, 1971. There exists a constant $C>0$ so that

$$
\sup _{u \in W_{0}^{1,2}(\Omega):\|\nabla u\|_{2} \leq 1} \int_{\Omega} \exp \left(\alpha u^{2}\right) d x \leq C|\Omega|, \quad \forall \alpha \leq 4 \pi .
$$

Moreover, $4 \pi$ is the best constant, that is, the supremum in (1.2) is $+\infty$ if $\alpha>4 \pi$. 
Estimate (1.2) is now referred as Trudinger-Moser inequality and plays an important role in geometric analysis and partial differential equations.

On the crucial question of compactness for the imbedding $W_{0}^{1,2}(\Omega) \hookrightarrow L_{\phi_{\alpha}}(\Omega)$ with $\phi_{\alpha}(t)=\exp \left(\alpha t^{2}\right)-1$, P. -L. Lions [16] proved that except for "small weak neighborhoods of 0 " the imbedding is compact and the best constant $4 \pi$ may be improved in a certain sense. More specifically, among other results, P. -L. Lions proved the following:

Lions, 1985. Let $\left(u_{k}\right)$ be a sequence of functions in $W_{0}^{1,2}(\Omega)$ with $\left\|\nabla u_{k}\right\|_{2}=1$ such that $u_{k} \rightarrow u \not \equiv 0$ weakly in $W_{0}^{1,2}(\Omega)$. Then for any $0<p<4 \pi /\left(1-\|\nabla u\|_{2}^{2}\right)$ we have

$$
\sup _{k} \int_{\Omega} \exp \left(p u_{k}^{2}\right) d x<\infty
$$

It is clear that this result gives more precise information than (1.2) when $u_{k} \rightarrow u$ weakly in $W_{0}^{1,2}(\Omega)$ with $u \not \equiv 0$ and it will be crucial to prove our main result. In this context, we are concerned about the existence of solution for $(P)$ when the nonlinearity $f(x, s)$ has the maximal growth on $s$ for which the functional $\Phi(u):=\int_{\Omega} F(x, u) \mathrm{d} x$, where $F(x, s)=\int_{0}^{s} f(x, t) \mathrm{d} t$, can be studied on the $W_{0}^{1,2}(\Omega)$-setting. To be more precise, following the lines of [1, 7, , 8] and motivated by the Trudinger-Moser inequality (1.2), we say that $f(x, s)$ has exponential subcritical growth at $+\infty$ if $\lim _{s \rightarrow+\infty} f(x, s) \exp \left(-\alpha s^{2}\right)=0$ for any $\alpha>0$ and $f(x, s)$ has exponential critical growth at $+\infty$, if there is $\alpha_{0}>0$ such that

$(c)_{\alpha_{0}}$

$$
\lim _{s \rightarrow+\infty} f(x, s) \exp \left(-\alpha s^{2}\right)=\left\{\begin{aligned}
0, & \forall \alpha>\alpha_{0}, \\
+\infty, & \forall \alpha<\alpha_{0},
\end{aligned}\right.
$$

uniformly in $x \in \Omega$. We will restrict our discussion for the case that $f(x, s)$ has exponential critical growth which is more involved.

For ease of reference we state our assumptions on $m$ and $f$ in a more precise way. For this, we define $M(t)=\int_{0}^{t} m(s) \mathrm{d} s$, the primitive of $m$ so that $M(0)=0$. The hypotheses on the function $m: \mathbb{R}_{+} \rightarrow \mathbb{R}_{+}$are the following:

$\left(M_{1}\right)$ there exists $m_{0}>0$ such that $m(t) \geq m_{0}$ for all $t \geq 0$ and

$$
M(t+s) \geq M(t)+M(s) \quad \forall s, t \geq 0 ;
$$

$\left(M_{2}\right)$ there exists constants $a_{1}, a_{2}>0$ and $t_{0}>0$ such that for some $\sigma \in \mathbb{R}$

$$
m(t) \leq a_{1}+a_{2} t^{\sigma}, \quad \forall t \geq t_{0}
$$

$\left(M_{3}\right) \frac{m(t)}{t}$ is nonincreasing for $t>0$.

Note that condition $\left(M_{1}\right)$ is valid whenever $m(0):=m_{0}>0$ and $m$ is nondecreasing. A typical example of a function $m$ satisfying the conditions $\left(M_{1}\right)-\left(M_{3}\right)$ is given by

$$
m(t)=m_{0}+a t
$$


where $m_{0}>0$ and $a \geq 0$, which is the model considered in the original Kirchhoff equation (1.1). An another example is $m(t)=1+\ln (1+t)$.

As a consequence of $\left(M_{3}\right)$ (see proof of Lemma 4.3), a straightforward computation shows that

$\left(\widehat{M}_{3}\right) \frac{1}{2} M(t)-\frac{1}{4} m(t) t$ is nondecreasing for $t \geq 0$.

In particular, one has

$$
\frac{1}{2} M(t)-\frac{1}{4} m(t) t \geq 0, \quad \forall t \geq 0 .
$$

Here, we also require that $f: \Omega \times \mathbb{R} \rightarrow \mathbb{R}$ is continuous. Since we intend to find positive solutions, in all this paper let us assume that $f(x, s)=0$ for $x \in \Omega$ and for $s \leq 0$. Moreover, $f$ satisfies $(c)_{\alpha_{0}}$ and the following conditions:

$\left(f_{1}\right)$ there exist constants $s_{0}, K_{0}>0$ such that

$$
F(x, s) \leq K_{0} f(x, s) \quad \forall(x, s) \in \Omega \times\left[s_{0},+\infty\right) ;
$$

$\left(f_{2}\right)$ for each $x \in \Omega, \frac{f(x, s)}{s^{3}}$ is increasing for $s>0$;

$\left(f_{3}\right)$ there exists $\beta_{0}>\frac{2}{\alpha_{0} d^{2}} m\left(4 \pi / \alpha_{0}\right)$ such that

$$
\lim _{s \rightarrow+\infty} \frac{s f(x, s)}{\exp \left(\alpha_{0} s^{2}\right)} \geq \beta_{0}, \quad \text { uniformly in } x \in \Omega,
$$

where $d$ is the radius of the largest open ball contained in $\Omega$.

We observe that condition $\left(f_{1}\right)$ implies

$$
F(x, s) \geq F\left(x, s_{0}\right) \exp \left[K_{0}\left(s-s_{0}\right)\right] \quad \forall(x, s) \in \mathbb{R}^{2} \times\left[s_{0},+\infty\right),
$$

which is reasonable for functions $f(x, s)$ behaving as $\exp \left(\alpha_{0} s^{2}\right)$ at infinity. Moreover, from $\left(f_{1}\right)$, for each $\theta>0$ there exists $R_{\theta}>0$ satisfying

$$
\theta F(x, s) \leq s f(x, s), \quad \forall(x, s) \in \Omega \times\left[R_{\theta},+\infty\right) .
$$

We also have that condition $\left(f_{2}\right)$ implies that

$$
\lim _{s \rightarrow 0^{+}} \frac{f(x, s)}{s^{\mu}}=0 \quad \text { uniformly in } x \in \Omega
$$

provided that $\mu \in[0,3)$. In particular, we have $f(x, 0)=0$ for each $x \in \Omega$. As we will see later on, hypothesis $\left(f_{3}\right)$ is necessary to obtain precise information about the minimax level of the energy functional associated to problem $(P)$.

Generally, the main difficulty encountered in nonlocal Kirchhoff problems is the competition that there is between the growths of $m$ and $f$. To overcome this trouble, the authors usually assume that $m$ is increasing or bounded, as we can 
see in [2, 4, 6, 9, 11, 14, 17, 20. We point out that in our arguments we do not suppose that $m$ is increasing and not bounded above. This allows us to consider the case $m(t) \equiv 1$ that corresponds to the Dirichlet problem

$$
\left\{\begin{array}{ccc}
-\Delta u=f(x, u) & \text { in } & \Omega, \\
u=0 & \text { on } & \partial \Omega .
\end{array}\right.
$$

Furthermore, for the authors knowledge, at the present time, there is no nonlocal problem involving critical growth of Trudinger-Moser type. For problems related to $(P)$, involving critical growth in the Sobolev case, we refer the papers [4, 12, 20].

We say that $u \in W_{0}^{1,2}(\Omega)$ is a weak solution of $(P)$ if holds

$$
m\left(\|u\|^{2}\right) \int_{\Omega} \nabla u \nabla \phi \mathrm{d} x=\int_{\Omega} f(x, u) \phi \mathrm{d} x, \quad \forall \phi \in W_{0}^{1,2}(\Omega) .
$$

Since $f(x, 0)=0, u \equiv 0$ is the trivial solution for $(P)$. Thus, our aim is to obtain a nontrivial solution. The term ground state refers to minimizers of the corresponding energy within the set of nontrivial solutions (see Section 2). Now, the main result of this work can state as follows.

Theorem 1.1. Suppose $\left(M_{1}\right)-\left(M_{3}\right),(c)_{\alpha_{0}}$ and $\left(f_{1}\right)-\left(f_{3}\right)$ are satisfied. Then, problem $(P)$ has a positive ground state solution.

An example of a function $f$ satisfying the conditions in Theorem 1.1, with $\alpha_{0}=1$, is given by

$$
F(x, s)=\frac{s^{4}}{4}+s^{2}\left[\exp \left(s^{2}\right)-1\right], s \geq 0 .
$$

Indeed, deriving we get

$$
f(x, s)=s^{3}+2 s\left[\exp \left(s^{2}\right)-1\right]+2 s^{3} \exp \left(s^{2}\right),
$$

from which one has $f(x, s) / s^{3}$ is increasing for $s>0$. A simple computation shows that

$$
\lim _{s \rightarrow+\infty} \frac{F(x, s)}{f(x, s)}=0 \quad \text { and } \quad \lim _{s \rightarrow+\infty} \frac{s f(x, s)}{\exp \left(s^{2}\right)}=+\infty,
$$

uniformly in $x \in \Omega$ and so $\left(f_{1}\right)-\left(f_{3}\right)$ are satisfied.

The paper is organized as follows. In Section 2 we present the variational setting in which our problem will be treated. Section 3 is devoted to show that the energy functional has the mountain pass geometry and in Section 4 we obtain an estimate for the minimax level associated to the our functional. Finally, we prove Theorem 1.1 in Section 5.

Hereafter, $C, C_{0}, C_{1}, C_{2}, \ldots$ will denote positive (possibly different) constants. We shall use the notation $\|\cdot\|$ for the norm of the gradient in the Sobolev space $W_{0}^{1,2}(\Omega)$ and $\|\cdot\|_{p}$ for the norm in the Leabegue space $L^{p}(\Omega), 1 \leq p<\infty$. The abbreviation a.e. will mean almost everywhere. 


\section{The variational framework}

As we are interested in positive solution, from now on we shall assume $f(x, s)=0$ for $x \in \Omega$ and $s \leq 0$. Since $f(x, s)$ is continuous and satisfies $(c)_{\alpha_{0}}$ and (1.6) , for $\varepsilon>0, \alpha>\alpha_{0}$ and $q \geq 0$, there exists $C=C(\varepsilon, \alpha, q)>0$ such that

$$
|F(x, s)| \leq \varepsilon s^{2}+C|s|^{q} \exp \left(\alpha s^{2}\right) \quad \forall(x, s) \in \Omega \times \mathbb{R} .
$$

This together with (1.2) yields $F(\cdot, u) \in L^{1}(\Omega)$ for all $u \in W_{0}^{1,2}(\Omega)$. Consequently, the functional

$$
I(u):=\frac{1}{2} M\left(\|u\|^{2}\right)-\int_{\Omega} F(x, u) \mathrm{d} x
$$

is well defined on $W_{0}^{1,2}(\Omega)$. Moreover, by standard arguments, $I \in C^{1}\left(W_{0}^{1,2}(\Omega), \mathbb{R}\right)$ with

$$
\left\langle I^{\prime}(u), \phi\right\rangle=m\left(\|u\|^{2}\right) \int_{\Omega} \nabla u \nabla \phi \mathrm{d} x-\int_{\Omega} f(x, u) \phi \mathrm{d} x, \quad u, \phi \in W_{0}^{1,2}(\Omega) .
$$

Hence, its critical points correspond to weak solutions of $(P)$, that is, $I$ is the EulerLagrange functional associated to $(P)$. We are interested in ground state solution $u$ for $(P)$ in the following sense: $u$ is positive and minimizes the energy functional $I$ within the set of nontrivial solutions of $(P)$.

\section{Mountain pass structure}

In order to achieve our existence result, we shall use the following version of the mountain pass theorem due to Ambrosetti and Rabinowitz [5], without the PalaisSmale condition:

Theorem 3.1. Let $E$ be a Banach space and $\Phi \in C^{1}(E ; \mathbb{R})$ with $\Phi(0)=0$. Suppose that there exist $\rho, \tau>0$ and $e \in E$ such

$$
\inf _{\|u\|=\rho} \Phi(u) \geq \tau \quad \text { and } \quad \Phi(e) \leq 0
$$

Then $\Phi$ possesses a Palais-Smale sequence at level c characterized as

$$
c:=\inf _{\gamma \in \Gamma} \max _{t \in[0,1]} \Phi(\gamma(t)) \geq \tau,
$$

where $\Gamma=\{\gamma \in C([0,1] ; E): \gamma(0)=0$ and $\gamma(1)=e\}$. $\Phi$.

The number $c$ is called mountain pass level or minimax level of the functional

In the sequel, we show that the functional $I$ has the mountain pass geometry, condition (3.1) above. This is proved in the next lemmas:

Lemma 3.2. Assume that conditions $\left(M_{1}\right),(c)_{\alpha_{0}}$ and $\left(f_{2}\right)$ hold. Then, there exist positive numbers $\rho$ and $\tau$ such that

$$
I(u) \geq \tau, \forall u \in W_{0}^{1,2}(\Omega) \quad \text { with } \quad\|u\|=\rho .
$$


Proof. By using (2.1), we get

$$
\int_{\Omega} F(x, u) \mathrm{d} x \leq \varepsilon \int_{\Omega}|u|^{2} \mathrm{~d} x+C \int_{\Omega}|u|^{q} \exp \left(\alpha u^{2}\right) \mathrm{d} x, u \in W_{0}^{1,2}(\Omega) .
$$

Here, let us consider $q>2$. From Sobolev imbedding and Hölder inequality, for $\|u\| \leq \rho_{1}$ we reach

$$
\begin{aligned}
\int_{\Omega} F(x, u) \mathrm{d} x & \leq \varepsilon C_{1}\|u\|^{2}+C\|u\|_{2 q}^{q}\left[\int_{\Omega} \exp \left(2 \alpha\|u\|^{2}(u /\|u\|)^{2}\right) \mathrm{d} x\right]^{1 / 2} \\
& \leq \varepsilon C_{1}\|u\|^{2}+C_{2}\|u\|^{q}\left[\int_{\Omega} \exp \left(2 \alpha \rho_{1}^{2}(u /\|u\|)^{2}\right) \mathrm{d} x\right]^{1 / 2} .
\end{aligned}
$$

Thus, if $\rho_{1} \leq \sqrt{2 \pi / \alpha}$, using the Trudinger-Moser inequality (1.2) and condition $\left(M_{1}\right)$ one has

$$
I(u) \geq\|u\|^{2}\left(\frac{m_{0}}{2}-\varepsilon C_{1}-C_{3}\|u\|^{q-2}\right) .
$$

Next, fix $\varepsilon>0$ so that $m_{0} / 2-\varepsilon C_{1}>0$. Hence, since $q>2$, choose $0<\rho \leq \rho_{1}$ verifying $m_{0} / 2-\varepsilon C_{1}-C_{3} \rho^{q-2}>0$. Consequently, if $\|u\|=\rho$ then $I(u) \geq \tau$, where

$$
\tau:=\rho^{2}\left(\frac{m_{0}}{2}-\varepsilon C_{1}-C_{3} \rho^{q-2}\right)>0,
$$

and the lemma is proved.

Lemma 3.3. Assume that conditions $\left(M_{2}\right)$ and $\left(f_{1}\right)$ hold. Then, there exists $e \in W_{0}^{1,2}(\Omega)$ with $I(e)<0$ and $\|e\|>\rho$.

Proof. First, we observe that for all $t \geq t_{0}$ condition $\left(M_{2}\right)$ implies that

$$
M(t) \leq \begin{cases}a_{0}+a_{1} t+\frac{a_{2}}{\sigma+1} t^{\sigma+1}, & \text { if } \sigma \neq-1, \\ b_{0}+a_{1} t+a_{2} \ln t, & \text { if } \sigma=-1,\end{cases}
$$

where $a_{0}=\int_{0}^{t_{0}} m(t) \mathrm{d} t-a_{1} t_{0}-a_{2} t_{0}^{\sigma+1} /(\sigma+1)$ and $b_{0}=\int_{0}^{t_{0}} m(t) \mathrm{d} t-a_{1} t_{0}-a_{2} \ln t_{0}$. On the other hand, taking $\theta>\max \{2,2 \sigma+2\}$ and using (1.5) one can see that there exist constants $C_{1}, C_{2}>0$ such that

$$
F(x, s) \geq C_{1} s^{\theta}-C_{2} \quad \forall(x, s) \in \Omega \times[0,+\infty) .
$$

Now, choose arbitrarily $u_{0} \in W_{0}^{1,2}(\Omega)$ with $u_{0} \geq 0$ in $\Omega$ and $\left\|u_{0}\right\|=1$. Thus, from (3.2) and (3.3), for all $t \geq t_{0}$ we reach

$$
I\left(t u_{0}\right) \leq\left\{\begin{aligned}
\frac{a_{0}}{2}+\frac{a_{1}}{2} t^{2}+\frac{a_{2}}{2 \sigma+2} t^{2 \sigma+2}-C_{1}\left\|u_{0}\right\|_{\theta}^{\theta} t^{\theta}+C_{2}|\Omega|, & \text { if } \sigma \neq-1, \\
\frac{b_{0}}{2}+\frac{a_{1}}{2} t^{2}+\frac{a_{2}}{2} \ln t-C_{1}\left\|u_{0}\right\|_{\theta}^{\theta} t^{\theta}+C_{2}|\Omega|, & \text { if } \sigma=-1,
\end{aligned}\right.
$$

from which we conclude that $I\left(t u_{0}\right) \rightarrow-\infty$ as $t \rightarrow+\infty$ provided that $\theta>$ $\max \{2,2 \sigma+2\}$. Hence, the result follows by considering $e=t_{*} u_{0}$ for some $t_{*}>0$ enough large. 


\section{Minimax estimates}

According to Lemmas 3.2 and 3.3 , let be

$$
c_{*}=\inf _{\gamma \in \Upsilon} \max _{t \in[0,1]} I(\gamma(t))>0,
$$

the minimax level of $I$, where $\Upsilon=\left\{\gamma \in C\left([0,1], W_{0}^{1,2}(\Omega)\right): \gamma(0)=0, I(\gamma(1))<0\right\}$. In order to get a more precise information about the minimax level $c_{*}$ obtained by Theorem [3.1, let us consider the following sequence $\widetilde{G}_{n}: \mathbb{R}^{2} \rightarrow \mathbb{R}$ of scaled and truncated Green's functions and also considered by Moser (see [8]):

$$
\widetilde{G}_{n}(x)=\frac{1}{\sqrt{2 \pi}} \begin{cases}(\log n)^{1 / 2}, & \text { if }|x| \leq \frac{1}{n} \\ \frac{\log \frac{1}{|x|}}{(\log n)^{1 / 2}}, & \text { if } \frac{1}{n} \leq|x| \leq 1 \\ 0, & \text { if }|x| \geq 1 .\end{cases}
$$

Let $x_{0} \in \Omega$ be such that the open ball $B_{d}\left(x_{0}\right)$ is contained in $\Omega$, where $d$ was given in $\left(f_{3}\right)$. It is standard verify that the functions

$$
G_{n}(x):=\widetilde{G}_{n}\left(\frac{x-x_{0}}{d}\right), x \in \Omega
$$

belongs to $H_{0}^{1}(\Omega),\left\|G_{n}\right\|=1$ and the support of $G_{n}$ is contained in $B_{d}\left(x_{0}\right)$. Furthermore, we have

Lemma 4.1. The following inequality holds

$$
\liminf _{n \rightarrow \infty} \int_{B_{d}\left(x_{0}\right)} \exp \left(4 \pi G_{n}^{2}\right) d x \geq 3 \pi d^{2} .
$$

Proof. By change of variable and using the definition of $\widetilde{G}_{n}$, we have

$$
\begin{aligned}
\int_{B_{d}\left(x_{0}\right)} \exp \left(4 \pi G_{n}^{2}\right) \mathrm{d} x & =d^{2} \int_{B_{\frac{1}{n}}(0)} \exp \left(4 \pi \widetilde{G}_{n}^{2}\right) \mathrm{d} y+d^{2} \int_{\frac{1}{n} \leq|y|<1} \exp \left(4 \pi \widetilde{G}_{n}^{2}\right) \mathrm{d} y \\
& =\pi d^{2}+2 \pi d^{2} \int_{\frac{1}{n}}^{1} \exp \left[\frac{2(\log (1 / r))^{2}}{\log n}\right] r \mathrm{~d} r \\
& =\pi d^{2}+2 \pi d^{2} \log n \int_{0}^{1} n^{2 s^{2}-2 s} \mathrm{~d} s
\end{aligned}
$$

where we also have used the change of variable $s=\log (1 / r) / \log n$ in the last integral. Next, since

$$
2 s^{2}-2 s \geq\left\{\begin{aligned}
-2 s, & \text { for } s \in[0,1 / 2] \\
2 s-2, & \text { for } s \in[1 / 2,1]
\end{aligned}\right.
$$


we get

$$
\begin{aligned}
\log n \int_{0}^{1} n^{2 s^{2}-2 s} \mathrm{~d} s & \geq \log n \int_{0}^{\frac{1}{2}} n^{-2 s} \mathrm{~d} s+\log n \int_{\frac{1}{2}}^{1} n^{2 s-2} \mathrm{~d} s \\
& =1-\frac{1}{n} .
\end{aligned}
$$

Using this estimate in (4.2) and passing to the limit, we obtain the desired inequality.

Finally, the next result provides the desired estimate for the level $c_{*}$.

Lemma 4.2. If conditions $\left(M_{1}\right)-\left(M_{2}\right)$ and $\left(f_{3}\right)$ hold, then

$$
c_{*}<\frac{1}{2} M\left(\frac{4 \pi}{\alpha_{0}}\right)
$$

Proof. Since $G_{n} \geq 0$ in $\Omega$ and $\left\|G_{n}\right\|=1$, as in the proof of Lemma 3.3, we have that $I\left(t G_{n}\right) \rightarrow-\infty$ as $t \rightarrow+\infty$. Consequently,

$$
c_{*} \leq \max _{t>0} I\left(t G_{n}\right), \forall n \in \mathbb{N} .
$$

Thus, it suffices to show that $\max _{t>0} I\left(t G_{n}\right)<\frac{1}{2} M\left(4 \pi / \alpha_{0}\right)$ for some $n \in \mathbb{N}$. Suppose, by contradiction, that

$$
\max _{t>0} I\left(t G_{n}\right) \geq \frac{1}{2} M\left(\frac{4 \pi}{\alpha_{0}}\right), \forall n \in \mathbb{N} .
$$

As $I$ possesses the mountain pass geometry, for each $n$ there exists $t_{n}>0$ such that

$$
I\left(t_{n} G_{n}\right)=\max _{t>0} I\left(t G_{n}\right) .
$$

From this and using that $F(x, s) \geq 0$ for all $(x, s) \in \Omega \times \mathbb{R}$ by (4.3) one has $M\left(t_{n}^{2}\right) \geq M\left(4 \pi / \alpha_{0}\right)$. By condition $\left(M_{1}\right), M:[0,+\infty) \rightarrow[0,+\infty)$ is a increasing bijection and so

$$
t_{n}^{2} \geq \frac{4 \pi}{\alpha_{0}}
$$

On the other hand,

from which we obtain

$$
\left.\frac{d}{d t} I\left(t G_{n}\right)\right|_{t=t_{n}}=0
$$

$$
m\left(t_{n}^{2}\right) t_{n}^{2}=\int_{\Omega} f\left(x, t_{n} G_{n}\right) t_{n} G_{n} \mathrm{~d} x \geq \int_{B_{d}\left(x_{0}\right)} f\left(x, t_{n} G_{n}\right) t_{n} G_{n} \mathrm{~d} x .
$$

By change of variable,

$$
\begin{aligned}
m\left(t_{n}^{2}\right) t_{n}^{2} & \geq d^{2} \int_{B_{1}(0)} f\left(x_{0}+d x, t_{n} \widetilde{G}_{n}\right) t_{n} \widetilde{G}_{n} \mathrm{~d} x \\
& \geq d^{2} \int_{B_{1 / n}(0)} f\left(x_{0}+d x, \frac{t_{n}}{\sqrt{2 \pi}}(\log n)^{1 / 2}\right) \frac{t_{n}}{\sqrt{2 \pi}}(\log n)^{1 / 2} \mathrm{~d} x .
\end{aligned}
$$


In view of (4.4), it follows that $(\log n)^{1 / 2} t_{n} / \sqrt{2 \pi} \rightarrow+\infty$ as $n \rightarrow \infty$. Hence, by $\left(f_{3}\right)$ given $\delta>0$ there exists $s_{\delta}>0$ such that

$$
f(x, s) s \geq\left(\beta_{0}-\delta\right) \exp \left(\alpha_{0} s^{2}\right), \quad \forall(x, s) \in \Omega \times\left[s_{\delta},+\infty\right) .
$$

So we obtain $n_{0} \in \mathbb{N}$ such that

$$
f\left(x_{0}+d x, \frac{t_{n}}{\sqrt{2 \pi}}(\log n)^{1 / 2}\right) \frac{t_{n}}{\sqrt{2 \pi}}(\log n)^{1 / 2} \geq\left(\beta_{0}-\delta\right) \exp \left(\alpha_{0} \frac{t_{n}^{2}}{2 \pi} \log n\right),
$$

for all $n \geq n_{0}$. Thus,

$$
\begin{aligned}
m\left(t_{n}^{2}\right) t_{n}^{2} & \geq\left(\beta_{0}-\delta\right) d^{2} \exp \left(\alpha_{0} \frac{t_{n}^{2}}{2 \pi} \log n\right) \frac{\pi}{n^{2}} \\
& =\left(\beta_{0}-\delta\right) \pi d^{2} \exp (-2 \log n) \exp \left(\alpha_{0} \frac{t_{n}^{2}}{2 \pi} \log n\right) \\
& =\left(\beta_{0}-\delta\right) \pi d^{2} \exp \left[2\left(\alpha_{0} \frac{t_{n}^{2}}{4 \pi}-1\right) \log n\right] .
\end{aligned}
$$

Note that, from $\left(M_{2}\right)$, we can conclude that

$$
\frac{m\left(t_{n}^{2}\right) t_{n}^{2}}{\exp \left[2\left(\alpha_{0} \frac{t_{n}^{2}}{4 \pi}-1\right) \log n\right]} \rightarrow 0 \quad \text { if } \quad t_{n} \rightarrow+\infty .
$$

Hence, from (4.7), $\left(t_{n}\right)$ must be bounded in $\mathbb{R}$. So, up to a subsequence, $t_{n} \rightarrow t_{0} \geq$ $\sqrt{4 \pi / \alpha_{0}}$. Moreover, using (4.7) again, we must have $\alpha_{0} \frac{t_{0}^{2}}{4 \pi}-1 \leq 0$ and therefore

$$
t_{n}^{2} \rightarrow \frac{4 \pi}{\alpha_{0}}
$$

At this point, following arguments as in [7] and [8] we are going to estimate (4.5) more exactly. For this, in view of (4.6), for $0<\delta<\beta_{0}$ and $n \in \mathbb{N}$ we set

$$
D_{n, \delta}:=\left\{x \in B_{d}\left(x_{0}\right): t_{n} G_{n}(x) \geq s_{\delta}\right\} \quad \text { and } \quad E_{n, \delta}:=B_{d}\left(x_{0}\right) \backslash D_{n, \delta} .
$$

Thus, by splitting the integral (4.5) on $D_{n, \delta}$ and $E_{n, \delta}$ and using (4.6), it follows that

$$
\begin{aligned}
m\left(t_{n}^{2}\right) t_{n}^{2} \geq & \left(\beta_{0}-\delta\right) \int_{B_{d}\left(x_{0}\right)} \exp \left(\alpha_{0} t_{n}^{2} G_{n}^{2}\right) \mathrm{d} x-\left(\beta_{0}-\delta\right) \int_{E_{n, \delta}} \exp \left(\alpha_{0} t_{n}^{2} G_{n}^{2}\right) \mathrm{d} x \\
& +\int_{E_{n, \delta}} f\left(x, t_{n} G_{n}\right) t_{n} G_{n} \mathrm{~d} x
\end{aligned}
$$

Since $G_{n}(x) \rightarrow 0$ for almost everywhere $x \in B_{d}\left(x_{0}\right)$ we have that the characteristic functions $\chi_{E_{n, \delta}}$ satisfy

$$
\chi_{E_{n, \delta}} \rightarrow 1 \text { a.e. in } B_{d}\left(x_{0}\right) \text { as } n \rightarrow \infty .
$$


Moreover, $t_{n} G_{n}<s_{\delta}$ in $E_{n, \delta}$. Thus, invoking the Lebesgue dominated convergence theorem we obtain

$$
\int_{E_{n, \delta}} \exp \left(\alpha_{0} t_{n}^{2} G_{n}^{2}\right) \mathrm{d} x \rightarrow \pi d^{2} \quad \text { and } \quad \int_{E_{n, \delta}} f\left(x, t_{n} G_{n}\right) t_{n} G_{n} \mathrm{~d} x \rightarrow 0 .
$$

Now, by using these convergences, (4.4), (4.8) and Lemma 4.1, passing to the limit in (4.9) we reach

$$
\begin{aligned}
m\left(\frac{4 \pi}{\alpha_{0}}\right) \frac{4 \pi}{\alpha_{0}} & \geq\left(\beta_{0}-\delta\right) \liminf _{n \rightarrow \infty} \int_{B_{d}\left(x_{0}\right)} \exp \left(4 \pi G_{n}^{2}\right) \mathrm{d} x-\left(\beta_{0}-\delta\right) \pi d^{2} \\
& \geq\left(\beta_{0}-\delta\right) 2 \pi d^{2}, \quad \forall \delta \in\left(0, \beta_{0}\right),
\end{aligned}
$$

and doing $\delta \rightarrow 0^{+}$we get $\beta_{0} \leq \frac{2}{\alpha_{0} d^{2}} m\left(4 \pi / \alpha_{0}\right)$, which contradicts $\left(f_{3}\right)$. Thus, the lemma is proved.

At this stage, we consider the Nehari manifold associated to the functional $I$, namely,

$$
\mathcal{N}:=\left\{u \in W_{0}^{1,2}(\Omega):\left\langle I^{\prime}(u), u\right\rangle=0, u \neq 0\right\}
$$

and the number $b:=\inf _{u \in \mathcal{N}} I(u)$. To compare the minimax level $c_{*}$ and $b$, we need the following lemma:

Lemma 4.3. If condition $\left(f_{2}\right)$ holds then, for each $x \in \Omega$,

$$
s f(x, s)-4 F(x, s) \text { is increasing for } s>0 .
$$

In particular, $s f(x, s)-4 F(x, s) \geq 0$ for all $(x, s) \in \Omega \times[0,+\infty)$.

Proof. Suppose $0<s<t$. For each $x \in \Omega$, we obtain

$$
\begin{aligned}
s f(x, s)-4 F(x, s) & =\frac{f(x, s)}{s^{3}} s^{4}-4 F(x, t)+4 \int_{s}^{t} f(x, \tau) \mathrm{d} \tau \\
& <\frac{f(x, t)}{t^{3}} s^{4}-4 F(x, t)+\frac{f(x, t)}{t^{3}}\left(t^{4}-s^{4}\right) \\
& =t f(x, t)-4 F(x, t)
\end{aligned}
$$

and this proves the lemma.

The next result is crucial in our arguments to prove the existence of a ground state solution for $(\underline{P})$.

Lemma 4.4. If $\left(M_{3}\right)$ and $\left(f_{2}\right)$ are satisfied then $c_{*} \leq b$.

Proof. Let $u$ be in $\mathcal{N}$ and define $h:(0,+\infty) \rightarrow \mathbb{R}$ by $h(t)=I(t u)$. We have that $h$ is differentiable and

$$
h^{\prime}(t)=\left\langle I^{\prime}(t u), u\right\rangle=m\left(t^{2}\|u\|^{2}\right) t\|u\|^{2}-\int_{\Omega} f(x, t u) u \mathrm{~d} x, \quad \forall t>0 .
$$


Since $\left\langle I^{\prime}(u), u\right\rangle=0$, that is, $m\left(\|u\|^{2}\right)\|u\|^{2}=\int_{\Omega} f(x, u) u \mathrm{~d} x$, we get

$$
h^{\prime}(t)=t^{3}\|u\|^{4}\left[\frac{m\left(t^{2}\|u\|^{2}\right)}{t^{2}\|u\|^{2}}-\frac{m\left(\|u\|^{2}\right)}{\|u\|^{2}}\right]+t^{3} \int_{\Omega}\left[\frac{f(x, u)}{u^{3}}-\frac{f(x, t u)}{(t u)^{3}}\right] u^{4} \mathrm{~d} x .
$$

We observe that $h^{\prime}(1)=0$ and by $\left(M_{3}\right)$ and $\left(f_{2}\right)$, it follows that $h^{\prime}(t) \geq 0$ for $0<t<1$ and $h^{\prime}(t) \leq 0$ for $t>1$. Hence,

$$
I(u)=\max _{t \geq 0} I(t u)
$$

Now, defining $g:[0,1] \rightarrow W_{0}^{1,2}(\Omega), g(t)=t t_{0} u$, where $t_{0}$ is such that $I\left(t_{0} u\right)<0$, we have $g \in \Upsilon$ and therefore

$$
c_{*} \leq \max _{t \in[0,1]} I(g(t)) \leq \max _{t \geq 0} I(t u)=I(u) .
$$

Since $u \in \mathcal{N}$ is arbitrary $c_{*} \leq b$ and the proof is complete.

Remark 4.5. We observe that if $m(t) \equiv K$ for some $K>0$, then the arguments in the previous lemma work if we suppose the condition $f(x, s) / s$ is increasing for $s>0$ holds instead of $\left(f_{2}\right)$. In this case, we would have $s f(x, s)-2 F(x, s)$ increasing for $s>0$ as the property in Lemma 4.3.

Remark 4.6. We recall that a solution $u_{0}$ of $(\underline{P})$ is a ground state if $I\left(u_{0}\right)=d:=$ $\inf _{u \in \mathcal{S}} I(u)$ where

$$
\mathcal{S}:=\left\{u \in W_{0}^{1,2}(\Omega): I^{\prime}(u)=0, u \neq 0\right\} .
$$

Since $c_{*} \leq b \leq d$, in order to obtain a ground state $u_{0}$ for $(P)$ it is enough to show that there is $u_{0} \in \mathcal{S}$ and $I\left(u_{0}\right)=c_{*}$.

\section{$5 \quad$ Proof of Theorem 1.1}

This section is devoted to the proof of our main result. For this purpose, we shall use the following result of convergence, whose proof can be found, for instance, in [7]:

Lemma 5.1. Suppose $\Omega$ is a bounded domain in $\mathbb{R}^{2}$. Let $\left(u_{n}\right)$ be in $L^{1}(\Omega)$ such that $u_{n} \rightarrow u$ in $L^{1}(\Omega)$ and let $f(x, s)$ be a continuous function. Then $f\left(x, u_{n}\right) \rightarrow f(x, u)$ in $L^{1}(\Omega)$ provided that $f\left(x, u_{n}\right) \in L^{1}(\Omega)$ for all $n$ and $\int_{\Omega}\left|f\left(x, u_{n}\right) u_{n}\right| d x \leq C$.

Proof of Theorem 1.1. By Lemmas 3.2 and 3.3 we can invoke Theorem 3.1 to obtain a sequence $\left(u_{n}\right)$ in $W_{0}^{1,2}(\Omega)$ verifying

$$
I\left(u_{n}\right) \rightarrow c_{*} \text { and } I^{\prime}\left(u_{n}\right) \rightarrow 0 .
$$

By using (1.5), with $\theta>4,\left(M_{1}\right)$ and (1.4) we obtain

$$
\begin{aligned}
C+\left\|u_{n}\right\| & \geq I\left(u_{n}\right)-\frac{1}{\theta}\left\langle I^{\prime}\left(u_{n}\right), u_{n}\right\rangle \\
& \geq\left(\frac{\theta-4}{4 \theta}\right) m_{0}\left\|u_{n}\right\|^{2}-C_{\theta}|\Omega|, \forall n \in \mathbb{N},
\end{aligned}
$$


for some $C>0$, where $C_{\theta}=\sup \left\{|f(x, s) s-\theta F(x, s)|:(x, s) \in \Omega \times\left[0, R_{\theta}\right]\right\}$. Hence $\left(u_{n}\right)$ is bounded in $W_{0}^{1,2}(\Omega)$ and, up to a subsequence, for some $u_{0} \in W_{0}^{1,2}(\Omega)$, one has

$$
\begin{aligned}
& u_{n} \rightarrow u_{0} \text { in } W_{0}^{1,2}(\Omega), \\
& u_{n} \rightarrow u_{0} \text { in } L^{p}(\Omega) \text { for } 1 \leq p<\infty .
\end{aligned}
$$

In particular, $u_{n}(x) \rightarrow u_{0}(x)$ for almost every $x \in \Omega$ and by (5.1) it also follows that $\int_{\Omega}\left|f\left(x, u_{n}\right) u_{n}\right| \mathrm{d} x$ is bounded. Thus, we can apply Lemma 5.1 to conclude that

$$
\int_{\Omega} f\left(x, u_{n}\right) \mathrm{d} x \rightarrow \int_{\Omega} f\left(x, u_{0}\right) \mathrm{d} x
$$

and therefore using $\left(f_{1}\right)$ and generalized Lebesgue dominated convergence theorem, we can see that

$$
\int_{\Omega} F\left(x, u_{n}\right) \mathrm{d} x \rightarrow \int_{\Omega} F\left(x, u_{0}\right) \mathrm{d} x .
$$

At this point, we affirm that $u_{0} \neq 0$. In fact, suppose by contradiction that $u_{0} \equiv 0$. Hence, $\int_{\Omega} F\left(x, u_{n}\right) \mathrm{d} x \rightarrow 0$ and so

$$
\frac{1}{2} M\left(\left\|u_{n}\right\|^{2}\right) \rightarrow c_{*}<\frac{1}{2} M\left(4 \pi / \alpha_{0}\right) .
$$

Thus, there exist $n_{0} \in \mathbb{N}$ and $\beta>0$ such that $\alpha_{0}\left\|u_{n}\right\|^{2}<\beta<4 \pi$ for all $n \geq n_{0}$. Now, choose $q>1$ close to 1 and $\alpha>\alpha_{0}$ close to $\alpha_{0}$ so that we still have $q \alpha\left\|u_{n}\right\|^{2}<\beta<4 \pi$. From this and by using (1.6), $(c)_{\alpha_{0}}$, Hölder inequality, (1.2) and (5.2) we get

$$
\begin{aligned}
\left|\int_{\Omega} f\left(x, u_{n}\right) u_{n} \mathrm{~d} x\right| & \leq C_{1} \int_{\Omega}\left|u_{n}\right|^{2} \mathrm{~d} x+C_{2} \int_{\Omega}\left|u_{n}\right| \exp \left(\alpha u_{n}^{2}\right) \mathrm{d} x \\
& \leq C_{1}\left\|u_{n}\right\|_{2}^{2}+C_{2}\left\|u_{n}\right\|_{\frac{q}{q-1}}\left(\int_{\Omega} \exp \left[q \alpha\left\|u_{n}\right\|^{2}\left(u_{n} /\left\|u_{n}\right\|\right)^{2}\right] \mathrm{d} x\right)^{\frac{1}{q}} \\
& \leq C_{1}\left\|u_{n}\right\|_{2}^{2}+C_{3}\left\|u_{n}\right\|_{\frac{q}{q-1}} \longrightarrow 0 \text { as } n \rightarrow \infty .
\end{aligned}
$$

Hence, since

$$
\left\langle I^{\prime}\left(u_{n}\right), u_{n}\right\rangle=m\left(\left\|u_{n}\right\|^{2}\right)\left\|u_{n}\right\|^{2}-\int_{\Omega} f\left(x, u_{n}\right) u_{n} \mathrm{~d} x
$$

and $\left\langle I^{\prime}\left(u_{n}\right), u_{n}\right\rangle \rightarrow 0$ it follows that $m\left(\left\|u_{n}\right\|^{2}\right)\left\|u_{n}\right\|^{2} \rightarrow 0$. Consequently by $\left(M_{1}\right)$ $\left\|u_{n}\right\|^{2} \rightarrow 0$ and therefore $I\left(u_{n}\right) \rightarrow 0$, what is absurd and thus we must have $u_{0} \neq 0$. Next, we will make some assertions.

Assertion 1. $u_{0}>0$ in $\Omega$.

Proof: As $\left(u_{n}\right)$ is bounded, up to a subsequence, $\left\|u_{n}\right\| \rightarrow \rho_{0}>0$. Moreover, condition $I^{\prime}\left(u_{n}\right) \rightarrow 0$ implies that

$$
m\left(\rho_{0}^{2}\right) \int_{\Omega} \nabla u_{0} \nabla v \mathrm{~d} x=\int_{\Omega} f\left(x, u_{0}\right) v \mathrm{~d} x, \quad \forall v \in W_{0}^{1,2}(\Omega) .
$$


Taking $v=-u_{0}^{-}$, where $u^{ \pm}=\max \{ \pm u, 0\}$, it follows that $\left\|u^{-}\right\|^{2}=0$ and so $u=u^{+} \geq 0$. Using the growth of $f$ and Trudinger-Moser inequality, $f\left(\cdot, u_{0}\right) \in L^{p}(\Omega)$ for all $1 \leq p<\infty$ and therefore by elliptic regularity $u_{0} \in W^{2, p}(\Omega)$ for all $1 \leq p<\infty$. Hence, by virtue of Sobolev imbedding $u_{0} \in C^{1, \gamma}(\bar{\Omega})$. Now, if we define $\Omega_{0}:=\left\{x \in \Omega: u_{0}(x)=0\right\}$ and we suppose $\Omega_{0} \neq \emptyset$ then since $f(x, s) \geq 0$ and by applying a Harnark inequality (see Theorem 8.20 in [13]) we can conclude that $\Omega_{0}$ is an open and closed of $\Omega$. The connectedness of $\Omega$ implies $\Omega_{0}=\Omega$ and so $u_{0} \equiv 0$, which is a contradiction. Thus, we must have $\Omega_{0}=\emptyset$, i.e., $u_{0}>0$ in $\Omega$.

Assertion 2. $m\left(\left\|u_{0}\right\|^{2}\right)\left\|u_{0}\right\|^{2} \geq \int_{\Omega} f\left(x, u_{0}\right) u_{0} \mathrm{~d} x$.

Proof: Suppose by contradiction that $m\left(\left\|u_{0}\right\|^{2}\right)\left\|u_{0}\right\|^{2}<\int_{\Omega} f\left(x, u_{0}\right) u_{0} \mathrm{~d} x$, that is, $\left\langle I^{\prime}\left(u_{0}\right), u_{0}\right\rangle<0$. Using $\left(M_{1}\right)$, (1.6) and Sobolev imbedding, we can see that $\left\langle I^{\prime}\left(t u_{0}\right), u_{0}\right\rangle>0$ for $t$ sufficiently small. Thus, there exists $\sigma \in(0,1)$ such that $\left\langle I^{\prime}\left(\sigma u_{0}\right), u_{0}\right\rangle=0$, i.e., $\sigma u_{0} \in \mathcal{N}$. Thus, according to $\left(\widehat{M}_{3}\right)$, Lemma 4.3, semicontinuity of norm and Fatou Lemma we obtain

$$
\begin{aligned}
c_{*} \leq b \leq I\left(\sigma u_{0}\right)= & I\left(\sigma u_{0}\right)-\frac{1}{4}\left\langle I^{\prime}\left(\sigma u_{0}\right), \sigma u_{0}\right\rangle \\
= & \frac{1}{2} M\left(\left\|\sigma u_{0}\right\|^{2}\right)-\frac{1}{4} m\left(\left\|\sigma u_{0}\right\|^{2}\right)\left\|\sigma u_{0}\right\|^{2} \\
& +\frac{1}{4} \int_{\Omega}\left[f\left(x, \sigma u_{0}\right) \sigma u_{0}-4 F\left(x, \sigma u_{0}\right)\right] \mathrm{d} x \\
< & \frac{1}{2} M\left(\left\|u_{0}\right\|^{2}\right)-\frac{1}{4} m\left(\left\|u_{0}\right\|^{2}\right)\left\|u_{0}\right\|^{2} \\
& +\frac{1}{4} \int_{\Omega}\left[f\left(x, u_{0}\right) u_{0}-4 F\left(x, u_{0}\right)\right] \mathrm{d} x \\
\leq & \liminf _{n \rightarrow \infty}\left[\frac{1}{2} M\left(\left\|u_{n}\right\|^{2}\right)-\frac{1}{4} m\left(\left\|u_{n}\right\|^{2}\right)\left\|u_{n}\right\|^{2}\right] \\
& +\liminf _{n \rightarrow \infty}\left[\frac{1}{4} \int_{\Omega}\left(f\left(x, u_{n}\right) u_{n}-4 F\left(x, u_{n}\right)\right) \mathrm{d} x\right] \\
\leq & \lim _{n \rightarrow \infty}\left[I\left(u_{n}\right)-\frac{1}{4}\left\langle I^{\prime}\left(u_{n}\right), u_{n}\right\rangle\right]=c_{*},
\end{aligned}
$$

which is absurd and the assertion is proved.

Assertion 3. $I\left(u_{0}\right)=c_{*}$.

Proof: By using (5.3) and semicontinuity of norm, we have $I\left(u_{0}\right) \leq c_{*}$. We are going to show that the case $I\left(u_{0}\right)<c_{*}$ can not occur. Indeed, if $I\left(u_{0}\right)<c_{*}$ then $\left\|u_{0}\right\|^{2}<\rho_{0}^{2}$. Moreover,

$$
\frac{1}{2} M\left(\rho_{0}^{2}\right)=\lim _{n \rightarrow \infty} \frac{1}{2} M\left(\left\|u_{n}\right\|^{2}\right)=c_{*}+\int_{\Omega} F\left(x, u_{0}\right) \mathrm{d} x
$$

which implies $\rho_{0}^{2}=M^{-1}\left(2 c_{*}+2 \int_{\Omega} F\left(x, u_{0}\right) \mathrm{d} x\right)$. Next, defining $v_{n}=u_{n} /\left\|u_{n}\right\|$ and 
$v_{0}=u_{0} / \rho_{0}$, we have $v_{n} \rightarrow v_{0}$ in $W_{0}^{1,2}(\Omega)$ and $\left\|v_{0}\right\|<1$. Thus, by (1.3)

$$
\sup _{n \in \mathbb{N}} \int_{\Omega} \exp \left(p v_{n}^{2}\right) d x<\infty, \quad \forall p<\frac{4 \pi}{1-\left\|v_{0}\right\|^{2}} .
$$

On the other hand, by Assertion 2, (1.4) and Lemma 4.3 one has

$$
I\left(u_{0}\right) \geq \frac{1}{2} M\left(\left\|u_{0}\right\|^{2}\right)-\frac{1}{4} m\left(\left\|u_{0}\right\|^{2}\right)\left\|u_{0}\right\|^{2}+\frac{1}{4} \int_{\Omega}\left[f\left(x, u_{0}\right) u_{0}-4 F\left(x, u_{0}\right)\right] \mathrm{d} x \geq 0 .
$$

Using this information together with Lemma 4.2 and the equality

$$
2 c_{*}-2 I\left(u_{0}\right)=M\left(\rho_{0}^{2}\right)-M\left(\left\|u_{0}\right\|^{2}\right),
$$

where we have used (5.5), we get

$$
M\left(\rho_{0}^{2}\right) \leq 2 c_{*}+M\left(\left\|u_{0}\right\|^{2}\right)<M\left(\frac{4 \pi}{\alpha_{0}}\right)+M\left(\left\|u_{0}\right\|^{2}\right)
$$

and therefore by $\left(M_{1}\right)$

$$
\rho_{0}^{2}<M^{-1}\left[M\left(\frac{4 \pi}{\alpha_{0}}\right)+M\left(\left\|u_{0}\right\|^{2}\right)\right] \leq \frac{4 \pi}{\alpha_{0}}+\left\|u_{0}\right\|^{2} .
$$

Since

$$
\rho_{0}^{2}=\frac{\rho_{0}^{2}-\left\|u_{0}\right\|^{2}}{1-\left\|v_{0}\right\|^{2}}
$$

from (5.7) it follows that

$$
\rho_{0}^{2}<\frac{\frac{4 \pi}{\alpha_{0}}}{1-\left\|v_{0}\right\|^{2}} .
$$

Thus, there exists $\beta>0$ such that $\alpha_{0}\left\|u_{n}\right\|^{2}<\beta<4 \pi /\left(1-\left\|v_{0}\right\|^{2}\right)$ for $n$ sufficiently large. For $q>1$ close to 1 and $\alpha>\alpha_{0}$ close to $\alpha_{0}$ we still have $q \alpha\left\|u_{n}\right\|^{2} \leq \beta<4 \pi /\left(1-\left\|v_{0}\right\|^{2}\right)$ and invoking (5.6) $)$, for some $C>0$ and $n$ large enough, we concluded that

$$
\int_{\Omega} \exp \left(q \alpha u_{n}^{2}\right) d x \leq \int_{\Omega} \exp \left(\beta v_{n}^{2}\right) d x \leq C .
$$

Hence, using (1.6), $(c)_{\alpha_{0}}$, Hölder inequality, (1.2) and (5.2) we reach

$$
\begin{aligned}
\left|\int_{\Omega} f\left(x, u_{n}\right)\left(u_{n}-u_{0}\right) \mathrm{d} x\right| & \leq C_{1} \int_{\Omega}\left|u_{n}-u\right|^{2} \mathrm{~d} x+C_{2} \int_{\Omega}\left|u_{n}-u_{0}\right| \exp \left(\alpha u_{n}^{2}\right) \mathrm{d} x \\
& \leq C_{1}\left\|u_{n}-u_{0}\right\|_{2}^{2}+C_{3}\left\|u_{n}-u_{0}\right\|_{\frac{q}{q-1}} \longrightarrow 0 \quad \text { as } \quad n \rightarrow \infty .
\end{aligned}
$$

Since $\left\langle I^{\prime}\left(u_{n}\right), u_{n}-u_{0}\right\rangle \rightarrow 0$, it follows that $m\left(\left\|u_{n}\right\|^{2}\right) \int_{\Omega} \nabla u_{n}\left(\nabla u_{n}-\nabla u_{0}\right) \mathrm{d} x \rightarrow 0$. On the other hand,

$$
\begin{gathered}
m\left(\left\|u_{n}\right\|^{2}\right) \int_{\Omega} \nabla u_{n}\left(\nabla u_{n}-\nabla u_{0}\right) \mathrm{d} x= \\
m\left(\left\|u_{n}\right\|^{2}\right)\left\|u_{n}\right\|^{2}-m\left(\left\|u_{n}\right\|^{2}\right) \int_{\Omega} \nabla u_{n} \nabla u_{0} \mathrm{~d} x \\
\longrightarrow m\left(\rho_{0}^{2}\right) \rho_{0}^{2}-m\left(\rho_{0}^{2}\right)\left\|u_{0}\right\|^{2},
\end{gathered}
$$


which implies that $\rho_{0}=\left\|u_{0}\right\|$. Thus, $\left\|u_{n}\right\| \rightarrow\left\|u_{0}\right\|$ and so $u_{n} \rightarrow u_{0}$. In view of the continuity of $I$, we must have $I\left(u_{0}\right)=c_{*}$ what is an absurd and the assertion is proved.

Finalizing the proof of Theorem [1.1: By Assertion 3 and (5.5) we can see that $M\left(\rho_{0}^{2}\right)=M\left(\left\|u_{0}\right\|^{2}\right)$ which shows that $\rho_{0}^{2}=\left\|u_{0}\right\|^{2}$. Hence, by (5.4) we have

$$
m\left(\left\|u_{0}\right\|^{2}\right) \int_{\Omega} \nabla u_{0} \nabla v \mathrm{~d} x=\int_{\Omega} f\left(x, u_{0}\right) v \mathrm{~d} x, \quad \forall v \in W_{0}^{1,2}(\Omega),
$$

that is, $u_{0}$ is a solution of $(P)$ satisfying $I\left(u_{0}\right)=c_{*}$ and according to Remark 4.6 and Assertion 1 the proof of our main result is complete.

Remark 5.2. As a matter of fact, by a slight modification of the previous proof, we can prove that the functional I satisfies the Palais-Smale condition at any level $c \in\left(-\infty, \frac{1}{2} M\left(4 \pi / \alpha_{0}\right)\right)$.

Remark 5.3. In line with Remark 4.5, we note that if $m(t) \equiv K$ then automatically the equality holds in the Assertion 1, and thus we would not need the condition $\left(f_{2}\right)$ to prove Assertion 1.

Acknowledgment Part of this work was done while the second author was visiting the Universidade Federal do Pará - UFPA. He would like to thank professor Giovany M. Figueiredo for his hospitality.

\section{References}

[1] Adimurthi, Existence of positive solutions of the semilinear Dirichlet problems with critical growth for the N-Laplacian, Ann. Sc. Norm. Sup. Pisa Cl. Sci. 17 (1990), 393-413.

[2] C. O. Alves, F. J. S. A. Corrêa and T. F. Ma, Positive solutions for a quasilinear elliptic equation of Kirchhoff type, Comput. Math. Appl. 49 (2005), 85-93.

[3] C. O. Alves and F. J. S. A. Corrêa, On existence of solutions for a class of problem involving a nonlinear operator, Comm. Appl. Nonlinear Anal. 8 (2001), $43-56$.

[4] C. O. Alves, F. J. S. A. Corrêa and G. M. Figueiredo, On a class of nonlocal elliptic problems with critical growth, Differ. Equ. Appl. 2 (2010), 409-417.

[5] A. Ambrosetti and P. H Rabinowitz, Dual variational methods in critical point theory and apllications, J. Functional Analysis 14 (1973), 349-381.

[6] B. Cheng, New existence and multiplicity of nontrivial solutions for nonlocal elliptic Kirchhoff type problems, J. Math. Anal. Appl. 394 (2012), 488-495.

[7] D. G. de Figueiredo, O. H. Miyagaki and B. Ruf, Elliptic equations in $\mathbb{R}^{2}$ with nonlinearities in the critical growth range, Calc. Var. 3 (1995) 139-153. 
[8] D. G. de Figueiredo, O. H. Miyagaki and B. Ruf, Corrigendum: "Elliptic equations in $\mathbb{R}^{2}$ with nonlinearities in the critical growth range", Calc. Var. 4 (1996) 203.

[9] X. M. He and W. M. Zou, Infinitely many positive solutions for Kirchhoff-type problems, Nonlinear Anal. 70 (2009), 1407-1414.

[10] G. Kirchhoff, Mechanik, Teubner, Leipzig, 1883.

[11] G. M. Figueiredo, Existence of positive solution for a Kirchhoff problem type with critical growth via truncation argument, preprint.

[12] G. M. Figueiredo and J. R. Santos Júnior, Multiplicity of solutions for a Kirchhoff equation with subcritical or critical growth, Differential Integral Equations 25 (2012), 853-868.

[13] D. Gilbarg and N. Trudinger, Elliptic Partial Differential Equations of Second Order, Springer-Verlag, Berlin, 1983.

[14] Y. Li, F. Li and J. Shi, Existence of a positive solution to Kirchhoff type problems without compactness conditions, J. Differential Equations 253 (2012), 2285-2294.

[15] J. -L. Lions, On some questions in boundary value problems of mathematical physics, North-Holland Math. Stud., 30, North-Holland, Amsterdam-New York, 1978.

[16] P. -L. Lions, The concentration-compactness principle in the calculus of variations. The limit case. I, Rev. Mat. Iberoamericana 1 (1985), 145-201.

[17] D. Liu and P. Zhao, Multiple nontrivial solutions to a p-Kirchhoff equation, Nonlinear Anal. 75 (2012), 5032-5038.

[18] J. Moser, A sharp form of an inequality by N. Trudinger, Ind. Univ. Math. J. 20 (1971), 1077-1092.

[19] N. S. Trudinger, On the imbedding into Orlicz spaces and some applications, J. Math. Mech. 17 (1967), 473-484.

[20] J. Wang, L. Tian, J. Xu and F. Zhang, Multiplicity and concentration of positive solutions for a Kirchhoff type problem with critical growth, J. Differential Equations 253 (2012), 2314-2351. 